\title{
Epidermal growth factor receptor mutation heterogeneity analysis of pulmonary sarcomatoid carcinoma successfully treated with erlotinib: A case report
}

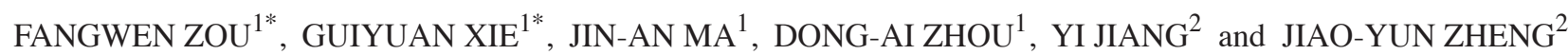 \\ Departments of ${ }^{1}$ Oncology and ${ }^{2}$ Pathology, The Second Xiangya Hospital of Central South University, \\ Changsha, Hunan 410011, P.R. China
}

Received May 29, 2014; Accepted February 2, 2015

DOI: $10.3892 / 01.2015 .3057$

\begin{abstract}
Pulmonary sarcomatoid carcinoma (PSC) is a rare histological subtype of non-small cell lung cancer, and the available studies on the response to epidermal growth factor receptor tyrosine kinase inhibitors (EGFR-TKIs) is limited. In the present study, a 73-year-old female presented with a large mass in the lower right lung, which was diagnosed as a PSC on biopsy. An amplification-refractory mutation system (ARMS) test revealed that the patient possessed the wild-type EGFR gene, and the patient subsequently underwent radiotherapy (60 Gy) and four 21-day cycles of chemoradiotherapy (1,600 mg gemcitabine, days 1 and $8 ; 30 \mathrm{mg}$, cisplatin, days 1-3). Following radiotherapy and chemotherapy treatment, a CT scan revealed complete remission of the mass in the lower right lung, however, metastases were identified in the paraaortic lymph node, bilateral iliac fossa and the right gluteal region. Notably, an EGFR exon 21 L858R gene mutation was identified in the mass of the right gluteal metastasis. Therefore, treatment with erlotinib was initiated. The patient continued to experience progression-free survival for six months following the initiation of erlotinib therapy. However, multiple metastases were then identified, and all lesions possessed the wild-type EGFR gene, as identified by the ARMS test. The findings suggest that erlotinib is a viable therapeutic option for the treatment of PSC patients that possess an EGFR mutation. The spatio-temporal evolution of EGFR mutational heterogeneity in PSC may result in drug-resistance, which challenges EGFR-TKI therapy and EGFR gene mutation diagnosis.
\end{abstract}

Correspondence to: Professor Jin-An Ma, Department of Oncology, The Second Xiangya Hospital of Central South University, 139 Renmin Road, Changsha, Hunan 410011, P.R. China E-mail: majinancs@hotmail.com

*Contributed equally

Key words: pulmonary sarcomatoid carcinoma, erlotinib, epidermal growth factor receptor mutation heterogeneity

\section{Introduction}

Pulmonary sarcomatoid carcinoma (PSC) is a rare and heterogeneous pulmonary tumor in which carcinomatous and sarcomatous elements occur, and accounts for $0.1-0.4 \%$ of all pulmonary malignant tumors (1). PSC predominantly affects males and smokers, with a median age of 51.4 years and a male to female ratio of 2.12-6:1 (2-4). Erlotinib, which is one of the highly promising epidermal growth factor receptor tyrosine kinase inhibitors (EGFR-TKIs), is administered orally once daily for the treatment of patients with non-small cell lung cancer (NSCLC). The present study reports the first case of PSC successfully treated with erlotinib. The spatio-temporal evolution of EGFR mutation heterogeneity between the primary lesion and metastasis, as well as between metastases, was also analyzed in the present study. Written informed consent was obtained from the patient's family.

\section{Case report}

A 73-year-old female was admitted to the Department of Oncology at Xiangya Second Hospital of Central South University (Changsha, China) in November 2012 due to violent coughing for 1 month. A computed tomography (CT) scan revealed a $5.2 \times 4.5 \mathrm{~cm}$ mass in the lower right lung (Fig. 1), and a pulmonary needle biopsy revealed carcinomatous and sarcomatous components within the lesion (Fig. 2A and B). Immunohistochemically, the tumor cells were positive for the expression of creatine kinase (CK; Fig. 2C), epithelial membrane antigen (EMA; Fig. 2D), vimentin (Fig. 2E) and Ki-67 (Fig. 2F), and did not express thyroid transcription factor-1 (TTF-1), smooth muscle actin (SMA) or S100. An amplification-refractory mutation system (ARMS) test revealed that the lesion possessed the wild-type EGFR gene. These findings were consistent with the features of PSC and clinical tumor-node-metastasis (cTNM) stage IIIa (cT3N2M0). The patient refused surgery and underwent four 21-day cycles of chemotherapy, comprising a regimen of gemcitabine (1,600 mg, days 1 and 8) and cisplatin (30 mg, days 1-3) combined with intensity-modulated radiotherapy (2 Gy/30 fractions). In December 2012, positron emission tomography/CT (Fig. 3) revealed that the right lower 


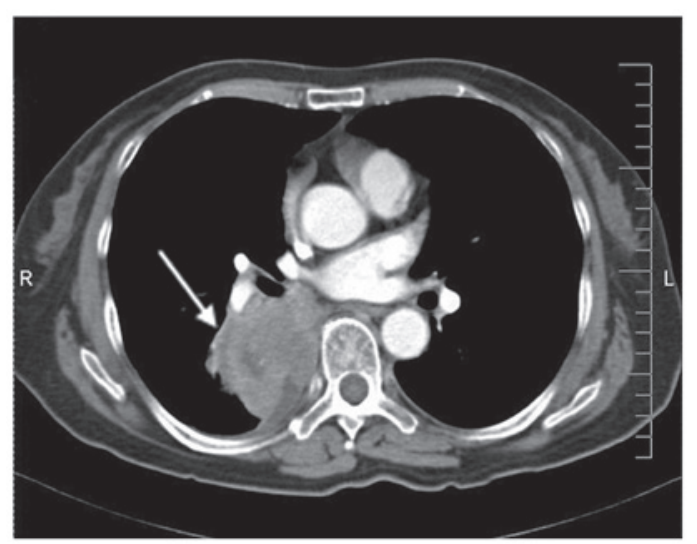

Figure 1. Computed tomography scan revealing the primary tumour to be $5.2 \times 4.5 \mathrm{~cm}^{2}$ in size and located in the right lower lung.

A

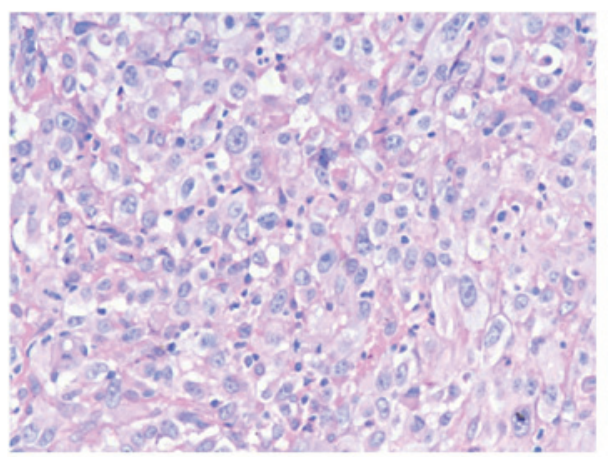

C

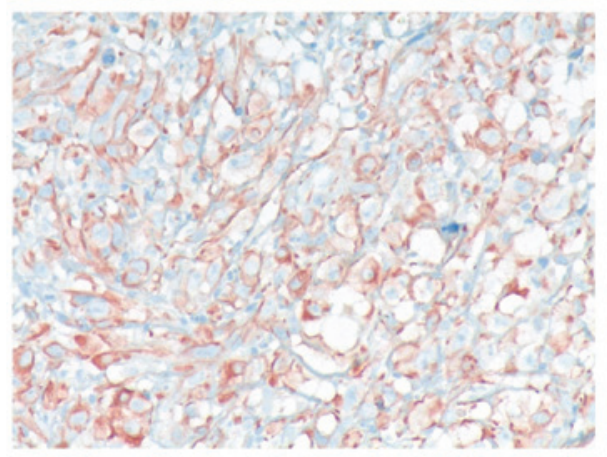

E

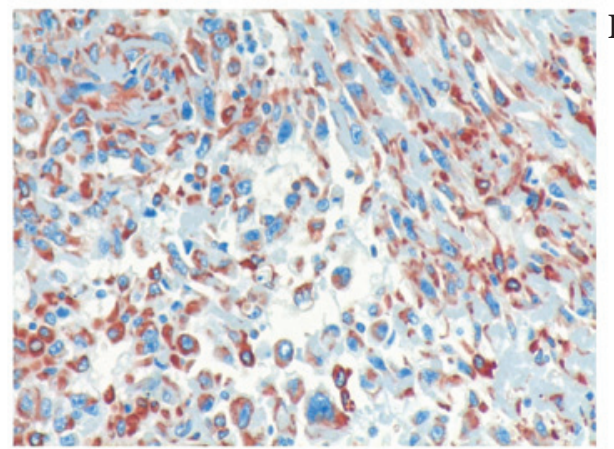

B

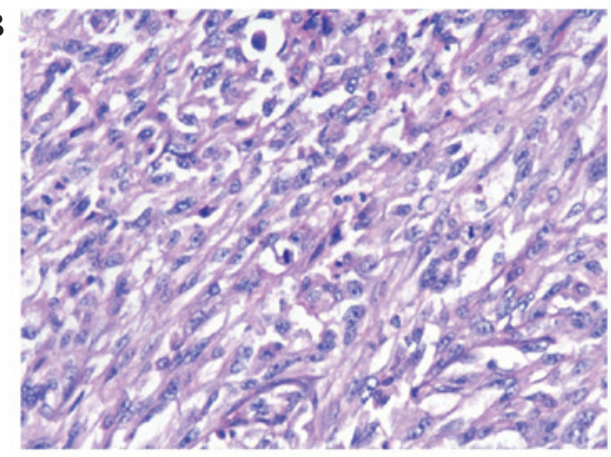

D
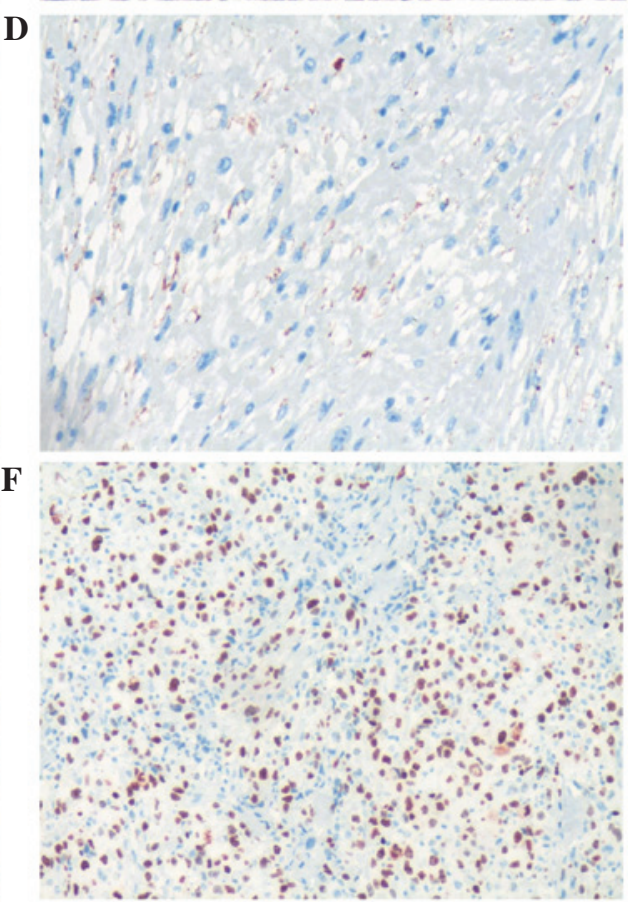

Figure 2. Histological analysis of the resected tumor specimen. (A) Carcinomatous component base. The tumor cells were irregular and pleomorphic, which can be observed in karyokinesis and necrosis tissues (HE stain; magnification, x200). (B) Sarcomatoid component base. The morphology of the tumor cells is varied, with the main form being immature and round or spindle-shaped cells. Certain cells possessed an indistinct cell boundary (HE stain; magnification, x200). Immunohistochemical staining of the resected tumor specimen demonstrating positive expression of (C) creatine kinase (++), (D) epithelial membrane antigen $(+),($ E) vimentin $(++)$ and $(\mathrm{F}) \mathrm{Ki}-67(++; 90 \%)$. HE, hematoxylin and eosin; (+), weakly positive staining (3-24\% cells positively stained); $(++)$, strongly positive staining $(25-49 \%$ cells positively stained).

mass of the lung was in complete remission, but metastases had arisen in the paraaortic lymph node, bilateral iliac fossa and the right gluteal region. Notably, an EGFR exon 21 L858R mutation was identified in the right gluteal metastatic lesion using the ARMS test. Therefore, treatment with $150 \mathrm{mg}$ of erlotinib once daily was initiated. However, six months subsequent 
A

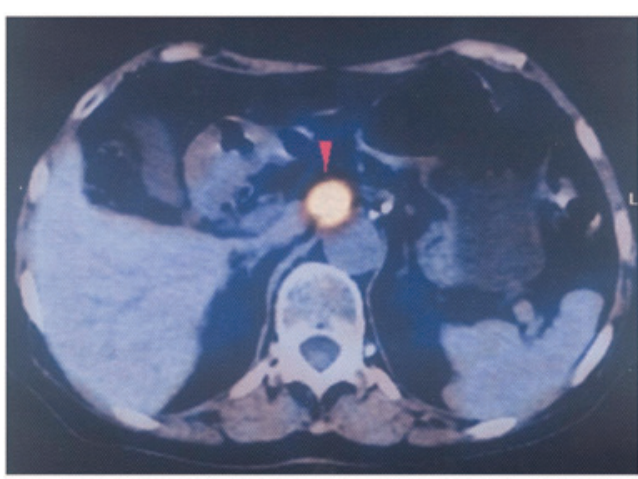

B
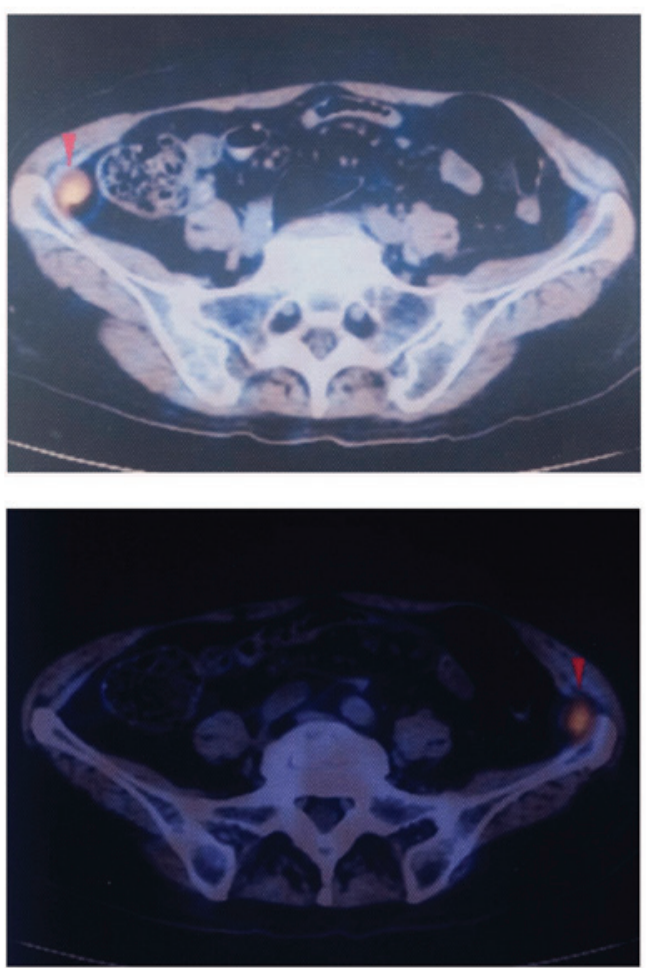

C

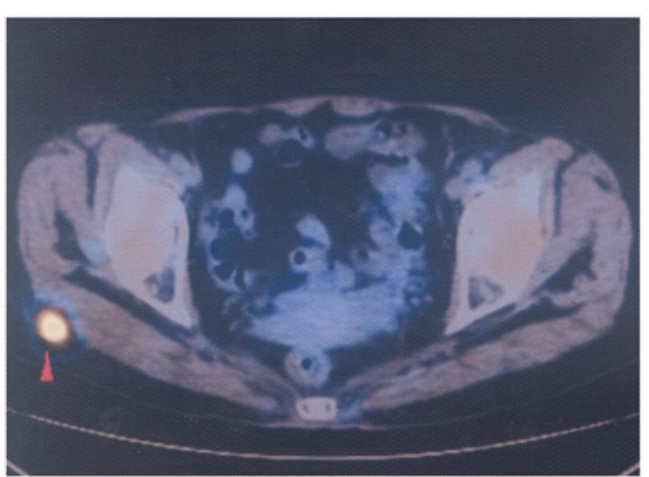

Figure 3. Positron emission tomography-computed tomography scan revealing the initial metastatic lesions in the (A) paraaortic lymph node (SUV value, 9.1), (B) bilateral left and right iliac fossa (SUV value, 4.7) and (C) right gluteal region (SUV value, 7.1). SUV, standardized uptake value.

to targeted therapy, CT (Fig. 4) revealed that the right iliac fossa metastases had decreased in size and the paraaortic lymph node metastasis had disappeared, but the left iliac fossa metastases had increased in size. Erlotinib was administered continually in combination with radiotherapy to treat the left iliac fossa metastasis. Two months later, a repeat CT (Fig. 5)

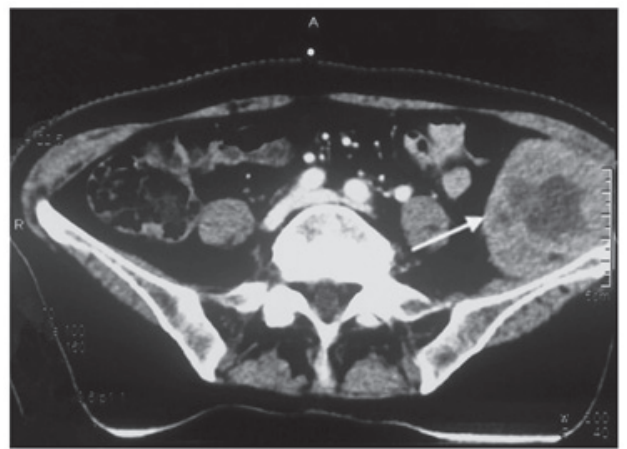

Figure 4. Computed tomography scan revealing metastases of the left iliac fossa to be $6 \times 5 \mathrm{~cm}$ in size, an increase compared with the previous size.

revealed that the left iliac fossa mass had decreased in size, but metastases were identified in the right gluteal region, right groin and the right chest wall of the right-lower abdomen. Biopsy of the right iliac fossa, right gluteal tumor and the right chest wall, the right abdominal subcutaneous nodules were all revealed to be metastatic sarcomatoid carcinoma, and all were revealed to also possess wild-type EGFR without the T790M mutation by performing an ARMS test (Fig. 6). The patient was therefore treated with two 21-day cycles of pemetrexed (800 mg, day 1), instead of erlotinib. In September 2013, CT re-examination revealed multiple organ metastases to the liver, pancreas and left adrenal gland. The patient received nutritional support, but succumbed to the disease in October 2013.

\section{Discussion}

According to the 2004 World Health Organization classification (5), PSC can be divided into five subtypes, consisting of pleomorphic carcinoma, spindle cell carcinoma, giant cell carcinoma, carcinosarcoma and pneumoblastoma. Overall, PSC runs an aggressive clinical course, with the five-year survival rate for patients with PSC being only $24.5 \%$, which is a worse rate compared with other forms of NSCLC $(6,7)$. In addition, distant metastasis can be identified in the early stages of the disease (8).

Surgery is currently considered to be the best option for the treatment of PSC (9) alongside chemoradiotherapy, which mainly refers to the chemotherapy regimens for NSCLC treatment (10). The effect of EGFR-TKIs on PSC remains unclear, and the available studies on the expression of EGFR are also limited. Italiano et al (11) tested the EGFR status of 22 PSC patients and the results revealed that none presented with EGFR mutation. However, Leone et al (12) retrospectively tested the EGFR status of 23 patients and the results revealed an EGFR exon 19 deletion in two patients. Ushiki et al (13) reported one patient who did not receive therapeutic effect from the administration of gefitinib. However, the autopsy of this patient revealed an EGFR exon 19 deletion, and a T790M drug-resistant mutation. In this case, the patient was a female with no smoking history. An EGFR exon 21 L858R mutation was identified in the metastatic lesion. In the present study, the patient experienced progression-free survival for the six months following the initiation of erlotinib therapy. However, resistance emerged finally, which may have been caused by EGFR mutational heterogeneity. 

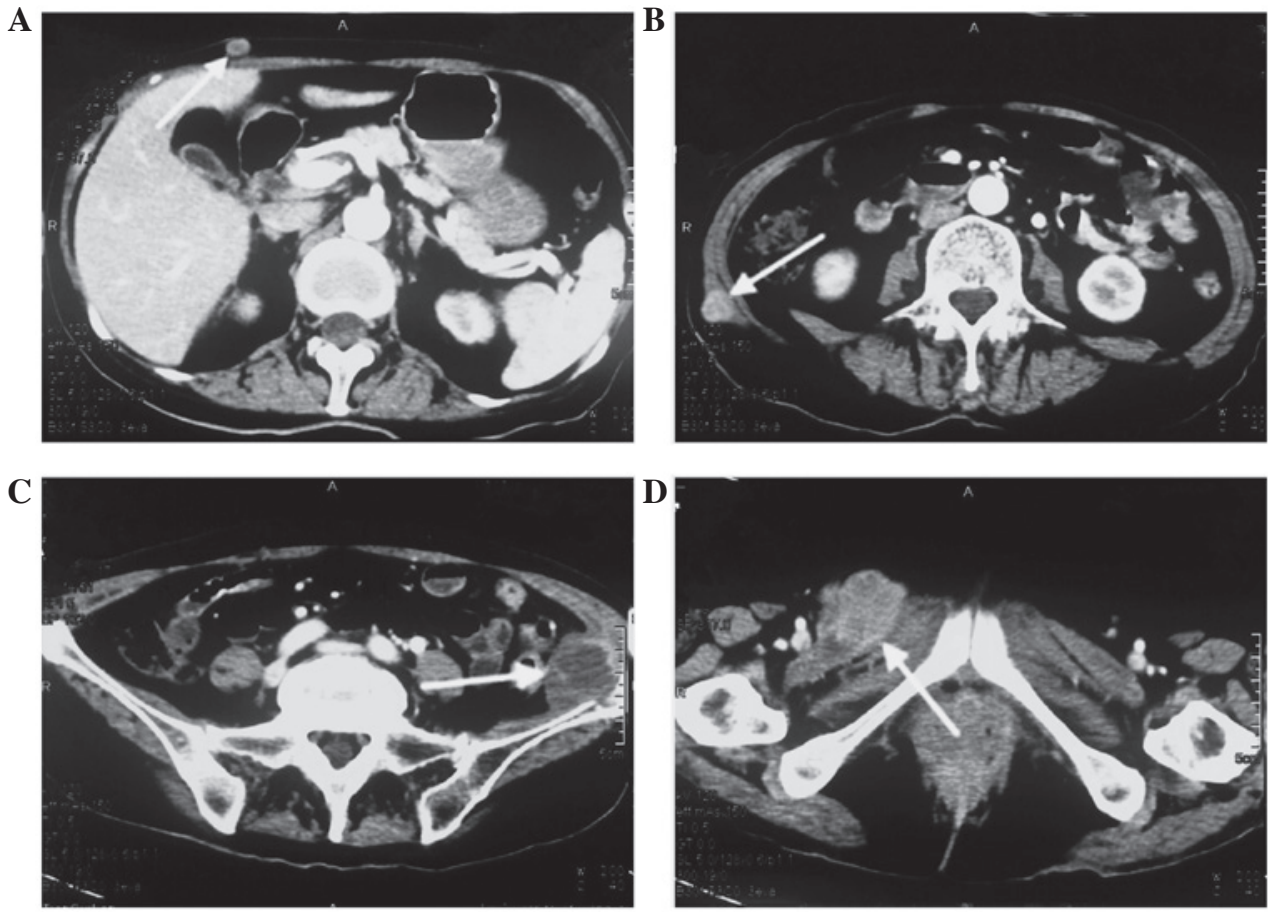

Figure 5. Computed tomography scan revealing the second group of metastatic lesions. Novel metastases were identified in (A) the right chest wall and (B) right lower abdomen. (C) The metastatic lesion in the left iliac fossa had decreased in size to $3.2 \times 3.6 \mathrm{~cm}$ in size. (D) A novel metastatic lesion, $4.5 \times 3.5 \mathrm{~cm}$ in size, was identified in the right gluteal region.
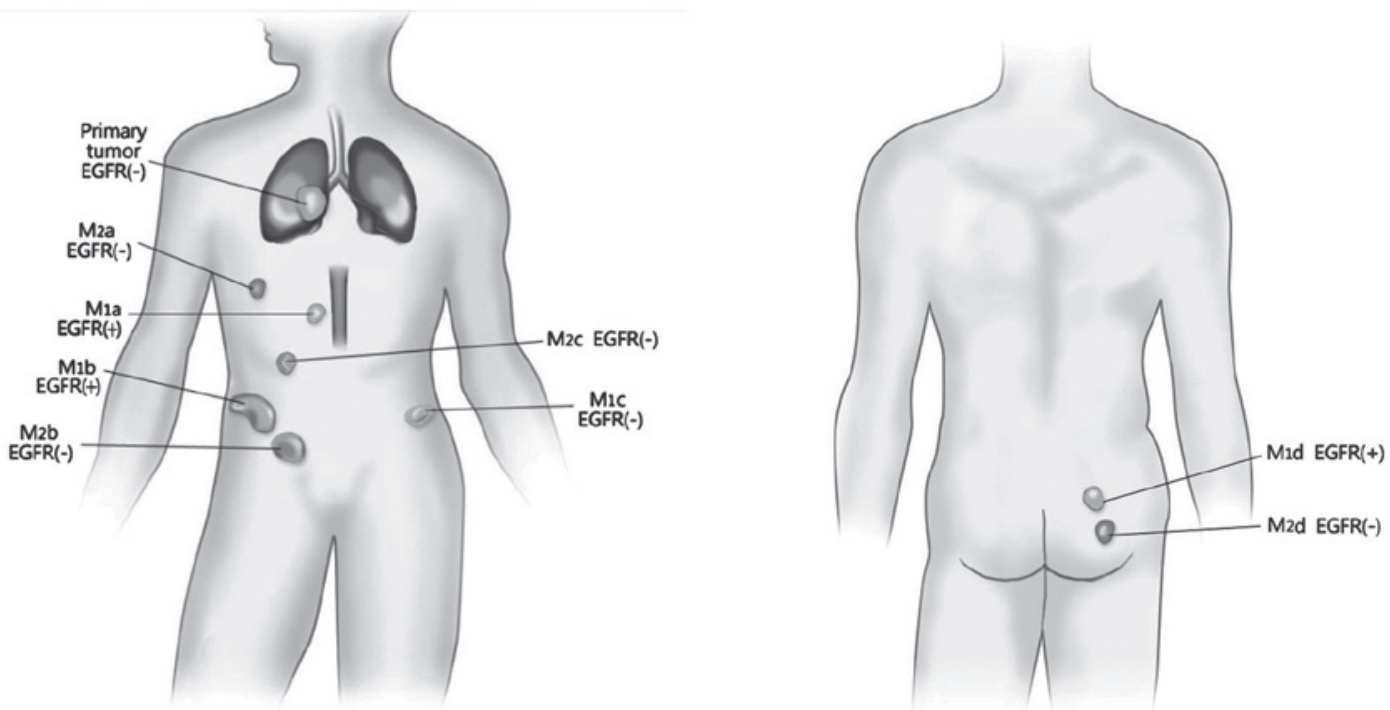

Figure 6. Diagram demonstrating the spatio-temporal evolution of the EGFR mutation. M1a, clinically-diagnosed EGFR(+) paraaortic lymph node lesion; M1b, clinicallay-diagnosed EGFR(+) lesion in the right iliohypogastric region; M1c, EGFR(-) lesion in the left iliohypogastric region, assessed by ARMS testing; and M1d, EGFR(+) lesion in the right gluteal region, assessed by ARMS testing. M2a, EGFR(-) lesion in the right chest wall, assessed by ARMS testing; M2b, EGFR(-) lesion in the right abdominal wall, assessed by ARMS testing; M2c, EGFR(-) lesion in the right groin, assessed by ARMS testing; and M2d, EGFR(-) lesion in the right gluteal region, assessed by ARMS testing. M1, inital group of metastatic lesions; M2, second group of metastatic lesions; EGFR, epidermal growth factor receptor; ARMS, amplification-refractory mutation system.

EGFR mutation heterogeneity is a common phenomenon in pulmonary carcinoma tissue $(14,15)$. Numerous studies have supported that the NSCLC primary and metastatic lesions also exhibit EGFR mutation heterogeneity (16-19). However, no study has yet reported EGFR mutation heterogeneity in PSC lesions. The present patient was identified as possessing EGFR mutation heterogeneity between the primary and metastatic lesions, as well as between the metastases, which demonstrated the double characteristics of temporal and spatial evolution. In the present study, the pulmonary primary lesion was EGFR wild-type, but in the first group of metastases, the lesion in the right gluteal region possessed a EGFR mutation and the lesion in the left iliac fossa was wild-type. Within the second group of metastases, the paraaortic lymph node and right iliac fossa 
metastases possessed EGFR mutations, according to the clinical response. Among the second array of metastases, all four lesions were wild type. With the exception of the pulmonary primary lesion, which was able to be well-controlled post-chemoradiotherapy, the therapeutic response of the metastatic lesions overall conformed to that expected due to the EGFR status. To the best of our knowledge, the spatio-temporal heterogeneous evolution of tumors has only been previously reported by Gerlinger et al (20) and Sequist et al (21).

In summary, the present study is the first case report of PSC successfully treated using erlotinib. The findings suggest that erlotinib is a viable therapeutic option for patients with PSC that possesses an EGFR mutation. However, the spatio-temporal evolution of EGFR mutational heterogeneity in PSC may result in drug-resistance, which challenges EGFR-TKI therapy and the diagnosis of EGFR gene mutation. Therefore, multiple biopsies may be necessary in cases exhibiting EGFR mutational heterogeneity.

\section{References}

1. Park JS, Lee Y, Han J, et al: Clinicopathologic outcomes of curative resection for sarcomatoid carcinoma of the lung. Oncology 11: 206-213, 2011

2. Zhou Z, Jiang GN, Yang TS, et al: Analysis for the prognosis of patients with pulmonary carcinosarcoma. Zhong Guo Ai Zheng Za Zhi 20: 59-61, 2010.

3. Fishback NF, Travis WD, Moran CA, et al: Pleomorphic (spindle/giant cell) carcinoma of the lung. A clinicopathologic correlation of 78 cases. Cancer 73: 2936-2945, 1994.

4. Nakajima M, Kasai T, Hashimoto H, et al: Sarcomatoid carcinoma of the lung: a clinicopathologic study of 37 cases. Cancer 86: 608-616, 1999

5. Beasley MB, Brambilla E and Travis WD: The 2004 World Health Organization classification of lung tumors. Semin Roentgenol 40: 90-97, 2005.

6. Venissac N, Pop D, Lassalle S, et al: Sarcomatoid lung cancer (spindle/giant cells): an aggressive disease? J Thorac Cardiovasc Surg 134: 619-623, 2007.

7. Brambilla E, Travis WD, Colby TV, Corrin B and Shimosato Y: The new World Health Organization classification of lung tumours. Eur Respir J 18: 1059-1068, 2001.

8. Homman T, Doki Y, Tsuda M, et al: Uncontrollable midbrain hemorrhage due to brain metastasis of pulmonary pleomorphic carcinoma. Kyobu Geka 61: 335-339, 2008 (In Japanese).
9. Petrov DB, Vlassov VI, Kalaydjiev GT, Plochev MA, Obretenov ED, Stanoev VI and Danon SE: Primary pulmonary sarcomas and carcinosarcomas - postoperative results and comparative survival analysis. Eur J Cardiothorac Surg 23: 461-466, 2003

10. Shi YK: NCCN clinical practice guidelines in non-small cell lung cancer V.2.2008. Zhong Hua Zhong Liu Za Zhi 30: 397-400, 2008.

11. Italiano A, Cortot AB, Ilie M, et al: EGFR and KRAS status of primary sarcomatoid carcinomas of the lung: implications for anti-EGFR treatment of a rare lung malignancy. Int J Cancer 125: 2479-2482, 2009.

12. Leone A, Graziano P, Gasbarra R, et al: Identification of EGFR mutations in lung sarcomatoid carcinoma. Int J Cancer 128: 732-735, 2011.

13. Ushiki A, Koizumi T, Kobayashi N, et al: Genetic heterogeneity of EGFR mutation in pleomorphic carcinoma of the lung: response to gefitinib and clinical outcome. Jpn J Clin Oncol 39: 267-270, 2009

14. Taniguchi K, Okami J, Kodama K, et al: Intratumor heterogeneity of epidermal growth factor receptor mutations in lung cancer and its correlation to the response to gefitinib. Cancer Sci 99: 929-935, 2008

15. Tomonaga N, Nakamura Y, Yamaguchi H, et al: Analysis of intratumor heterogeneity of EGFR mutations in mixed-type lung adenocarcinoma. Clin Lung Cancer 14: 521-526, 2013.

16. Gow CH, Chang YL, Hsu YC, et al: Comparison of epidermal growth factor receptor mutations between primary and corresponding metastatic tumors in tyrosine kinase inhibitor-naive non-small-cell lung cancer. Ann Oncol 20: 696-702, 2009.

17. Schmid K, Oehl N, Wrba F, et al: EGFR/KRAS/BRAF mutations in primary lung adenocarcinomas and corresponding locoregional lymph node metastases. Clin Cancer Res 15: 4554-4560, 2009.

18. Sun L, Zhang Q, Luan H, et al: Comparison of KRAS and EGFR gene status between primary non-small cell lung cancer and local lymph node metastases: implications for clinical practice. J Exp Clin Cancer Res 30: 30, 2011

19. Fang Q, Zhang L, Wang S and Ou W: Discordance of epidermal growth factor receptor mutations between primary and corresponding metastatic tumors in non-small cell lung cancer. Zhongguo Fei Ai Za Zhi 14: 518-522, 2011 (In Chinese).

20. Gerlinger M, Rowan AJ, Horswell S, et al: Intratumor heterogeneity and branched evolution revealed by multiregion sequencing. N Engl J Med 366: 883-892, 2012.

21. Sequist LV, Waltman BA, Dias-Santagata D, et al: Genotypic and histological evolution of lung cancers acquiring resistance to EGFR inhibitors. Sci Transl Med 3: 75ra26, 2011. 